\title{
Assessment of virtual thermal manikins for thermal comfort numerical studies. Verification and validation
}

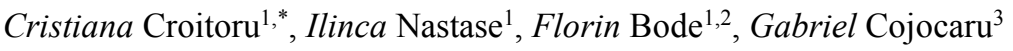 \\ ${ }^{1}$ CAMBI Research Centre, Faculty of Building Services Engineering, Technical University of Civil Engineering Bucharest, Romania \\ ${ }^{2}$ Technical University of Cluj Napoca, Romania \\ ${ }^{3}$ Renault Technologie Roumanie, Bucharest, Romania
}

\begin{abstract}
This study is a part of a larger experimental and numerical campaign intended to evaluate the influence of the turbulence intensity at the inlet of the terminal air distribution systems on the local draft sensation and thermal discomfort of ventilation users. In this paper we present preliminary results of CFD simulations using a realistic model of human body along with an experimental validation. The model is further used in a piston distribution scheme to evaluate the influence of turbulence intensity on the comfort indicators. The recorded velocity, turbulence and temperature fields allowed us to estimate the distributions of DR, PPD and PMV indexes. For the investigated case, the results indicated a direct correlation.
\end{abstract}

\section{Introduction}

Heating Ventilating Air Conditioning (HVAC) systems have to ensure clean air and to maintain comfortable conditions for their users as in the industrialised countries people spend up to $90 \%$ of their time indoors [1]. The recent energy crisis as well as the emergent awareness of the European leaders concerning environmental issues, are both lately sources of pressure on the EU members shoulders, which are supposed to to reduce their energy consumption within $20 \%$ before 2020 [2]. The reduced air mass flows being introduced in the occupied zone, should uniformly distribute fresh air and conditioning cooling or heating loads in order to achieve thermal comfort and acceptable air quality. Thus, innovative diffusers should be designed to improve mixing. In this context, an innovative air diffuser has to generate a strong induction, particularly in the initial region of the cold or hot air jet. The improved mixing will logically lead to a more stable flow and to a uniform distribution of the thermal load and will improve also the air quality. But this is not sufficient if an adequate air diffusion strategy is not implemented in the same time [3].

Therefore, numerical investigations such as those accomplished by CFD have been gaining immense popularity within the HVAC industry since the past few decades. A typical problem regarding this kind of numerical approach is that for the conception of building systems and simulation of the resulting indoor environment, it is still not acknowledged that convection flows caused by heat sources like the human body plume may significantly affect the flow distribution in rooms [4]. Generally, attention is given only on the flow generated by the air diffusion terminal devices. As shown by Kosonen et al [4] the point of occurrence of the maximum air velocity in the occupied zone depends on the heat source strength and its distribution in the room. Thus, the air flows interaction itself is of great importance when estimating occupants' comfort. In the same time, results obtained from computational fluid dynamics (CFD) need to be validated with experimental data from real scale measurements before using CFD for larger parametric investigation.

In the following we will pass briefly in review some of the main results concerning our studies of the human body environment and the related discussion about thermal comfort. Due to the lack of space, we will focus on the numerical approach (CFD) and some of the associated results.

This study is a part of a larger experimental and numerical campaign which is intended among other directions to study the influence of the turbulence intensity at the exit plane of the terminal air diffusion devices on the local draft sensation and thermal discomfort of mixing and personalized ventilation users.

\section{Methods}

During our research study we ran CFD simulations using a realistic model of human body along with an experimental validation. The thermal manikin used during the experimental approach was manufactured in our laboratory. It has a realistic shape and six differently heated surfaces [5]. In this study, PIV measurements were performed for validation. These measurements targeted experimental validation of the velocity distribution obtained by numerical study of convective flow above the manikin's head. PIV measurement campaigns were carried out using a Dantec system

\footnotetext{
* Corresponding author: cristiana.croitoru@utcb.ro
} 
composed of a $4 \mathrm{M}$ FlowSense MKII CCD camera of $4 \mathrm{x}$ $10^{6}$ pixels and a Litron of $200 \mathrm{~mJ}$ laser. The maximum acquisition frequency of the PIV system was $7.5 \mathrm{~Hz}$. During the experimental campaign, the surface temperature of the manikin was measured with sensors placed on the body and a FLIR B620 infrared camera, which was also used for evaluating the thermal plume above the head, using a thin black carboard. Also, temperature profile was measured above the head with PT 100 sensors.

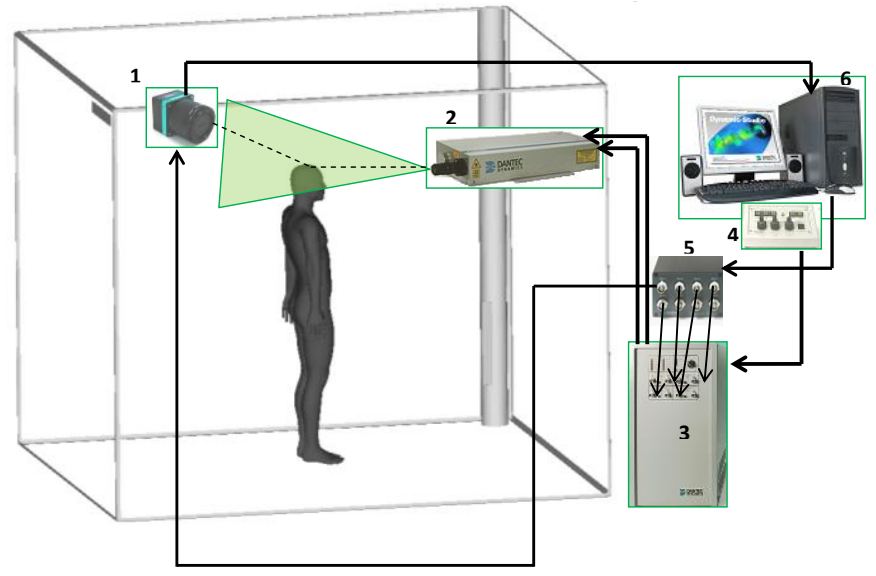

Fig. 1. Experimental setup.

The numerical model has been built after the experimental setup, with the same geometry of the manikin and room geometry. Thermal manikins (virtual and experimental) have 6 zones as indicated below:
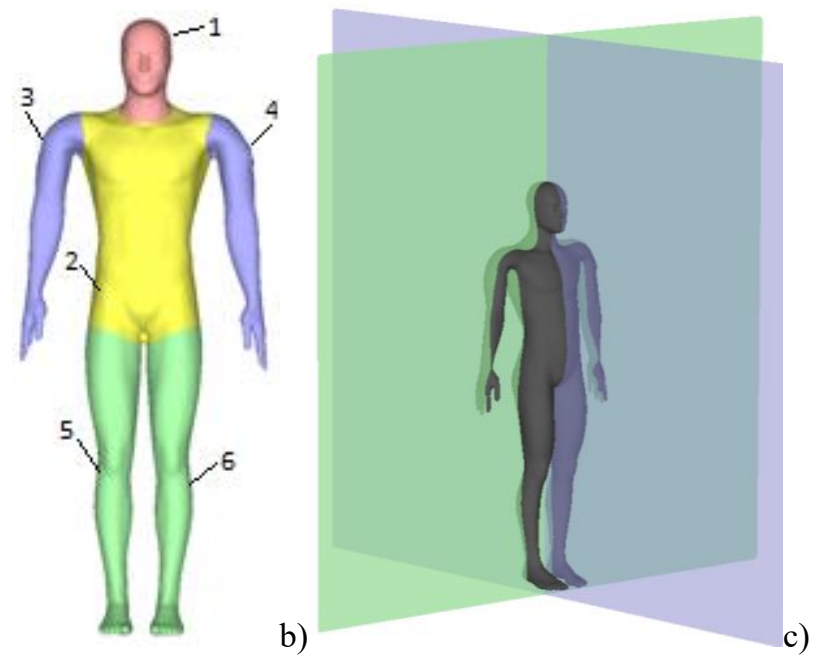

Fig. 2. Thermal manikin-details: a) 6 zones of the manikin: 1head, 2-torso, 3-right arm, 4-left arm, 5-right leg, 6-left leg b) sagittal plan (purple) and coronal (green)

\subsection{Grid choice}

Determining the required number of cells to calculate the solution is achieved through a study of independence of the solution to the number of mesh elements. This study is necessary as part of the verification process for the numerical simulation results in order to achieve a mesh independent solution. In a regular case, from a certain number of cells, the difference between the numerical results does not vary so much so that the choice of a computing grid is due to compromise solution between accuracy and computational resources.
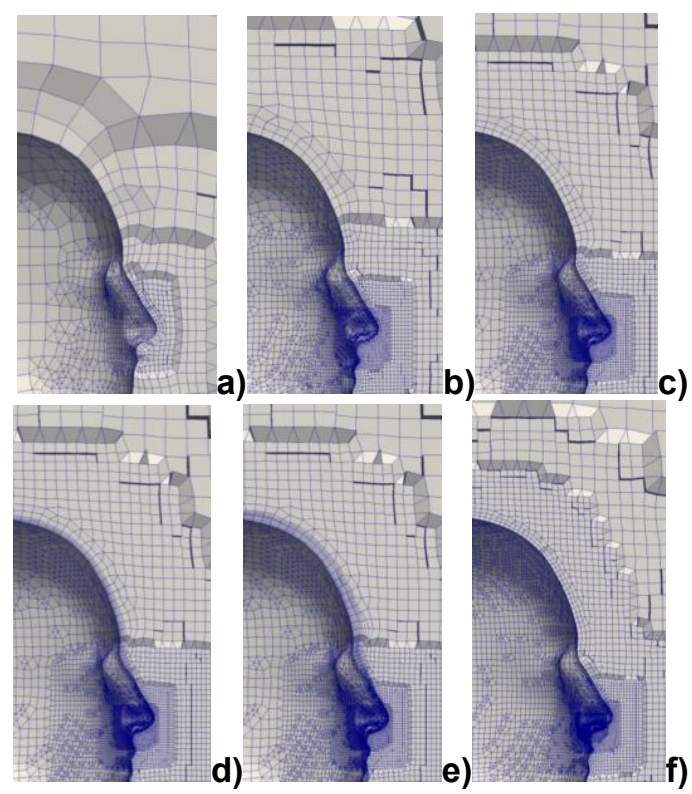

Fig. 3. Meshing level for the studied cases: a)0.2, b)0.6, c)1.2, d) 1.6, e) 2.2, f) 2.8 million cells

For the study of solution independency in function of the chosen mesh, we decided to choose a turbulence model which seems the most suitable for further use. Based on recent works, SST k-omega turbulence model is proving to be the most reliable of the two equation models when desiring reproduction of relatively complex flow, characterized by relatively low values of Reynolds number, as the convection current generated by the body and a jet of air used for ventilation [6]. So, we have chosen this model for the numerical approach and testing spatial mesh was made for this turbulence model. For our case, we made calculations respectively for $0.2,0.6$, $1.2,1.6,2.2$ and 2.8 million cells.

For this study natural convection case was chosen, without any inlet or outlet because by that time experimental validation measurements were made only in this case. We compared the velocity profiles overhead and velocity and temperature fields.

Simulations were performed for the case without jet in an ambient of $20^{\circ} \mathrm{C}$ for which the boundary conditions can be seen in the table below:

Table 2: Boundary conditions for the seven cases

\begin{tabular}{|c|}
\hline Boundary limits \\
\hline Inlet: Pressure inlet, $\mathrm{p}=0, \mathrm{t}=20^{\circ} \mathrm{C}$ \\
\hline Outlet: Pressure outlet, $\mathrm{p}=0, \mathrm{t}=20^{\circ} \mathrm{C}$ \\
\hline Head $\mathrm{t}=34.2^{\circ} \mathrm{C}$ \\
\hline Torso $\mathrm{t}=31.9^{\circ} \mathrm{C}$ \\
\hline Arms $\mathrm{t}=30^{\circ} \mathrm{C}$ \\
\hline Legs $\mathrm{t}=26.8^{\circ} \mathrm{C}$ \\
\hline Walls $\mathrm{t}=20^{\circ} \mathrm{C}$ \\
\hline
\end{tabular}

To check the mesh quality, we considered for each case, the minimum value of $y+$ on the surface. 
Below are the temperature fields in sagittal and coronal plan. We see the same trend of stabilization from the case of 1.2 million elements. The velocity fields indicate a stabilisation from 1.6 million elements.
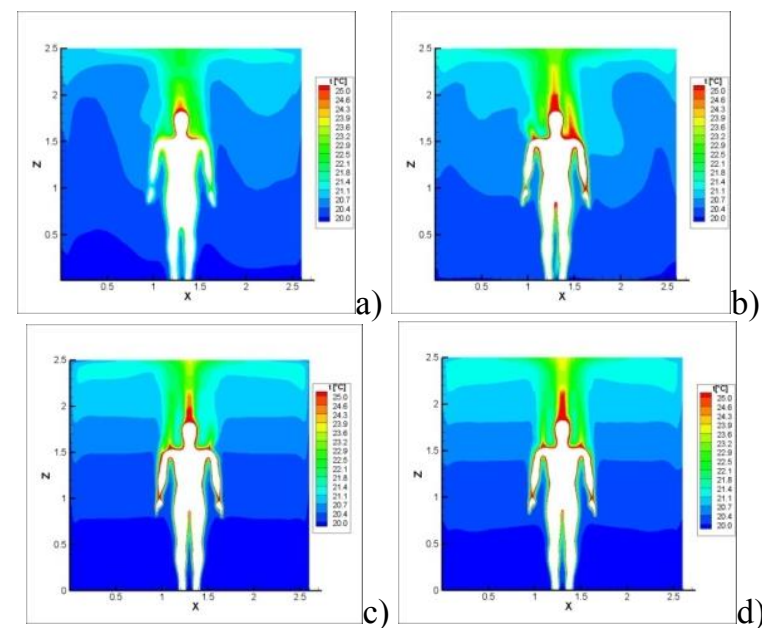

)
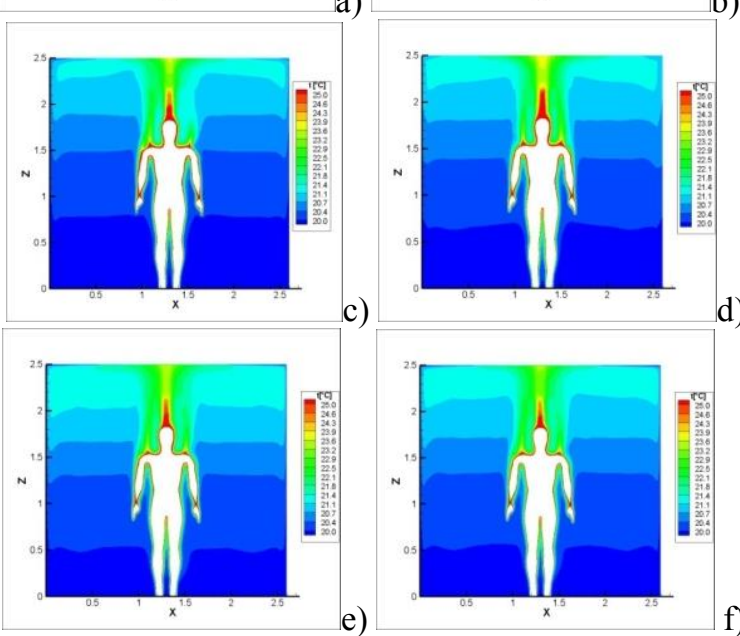

Fig. 4. Velocity fields-sagittal plan: a)0.2, b)0.6, c)1.2, d) 1.6, e) 2.2, f) 2.8 million cells

We followed in particular the thermal stratification phenomenon, recirculation zones formed by the impact of convection current and the ceiling of the test cell. Thus, we find that from 2.2 million cells we have no longer visible variation on velocity and temperature fields. We choose the geometry of 2.2 million cells to be used on the rest of the cases studied, because it qualifies as independent mesh solution.

\subsection{Turbulence model choice}

Once chosen the final mesh, we decided to check whether our choice oriented to SST k- $\omega$ turbulence model was appropriate, in other words to validate this choice of turbulence model by comparing the results for velocity and temperature profiles for seven turbulence models and reference data from PIV measurements and IR thermography. Turbulence models tested are respectively: k-epsilon standard, k-epsilon realizable, RNG k-epsilon, k-omega standard, SST k-omega, laminar and Spalart - Allmaras. Fluent solver gives a choice of several "upwind" interpolation schemes, the one used in this study is "second order upwind" to calculate the convective terms. Pressure-velocity coupling scheme chosen is given by the "SIMPLE" algorithm of the solver.

The validation of the model was accomplished by extensive experimental campaign with PIV measurements, IR thermography and vertical temperature measurements above the real manikin head.
Because the natural convective flow is the one that raises most of the problems [3, 7-9], this comparison was made for the case with no other flow than the convective one.
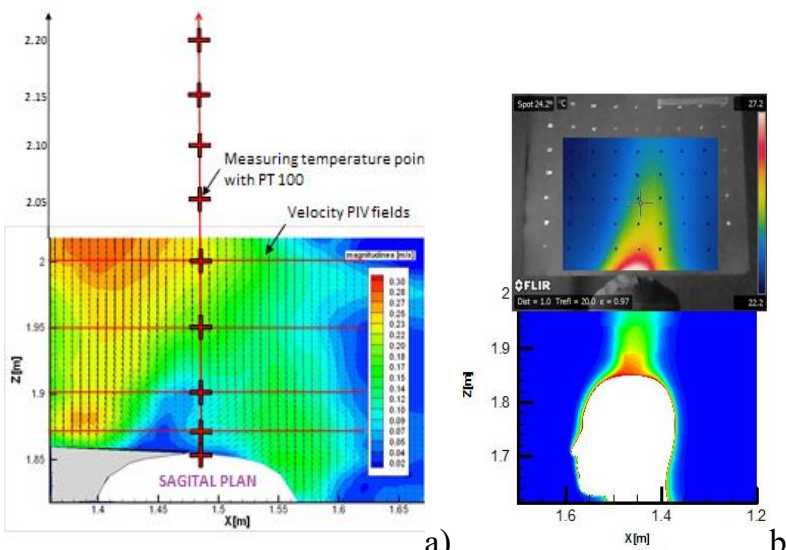

Fig. 5. a) Velocity field obtained by PIV measurements and position for the measured points for temperature; b) Comparison between temperature fields obtained by IR measurements and numerical simulation results with SST k- $\omega$
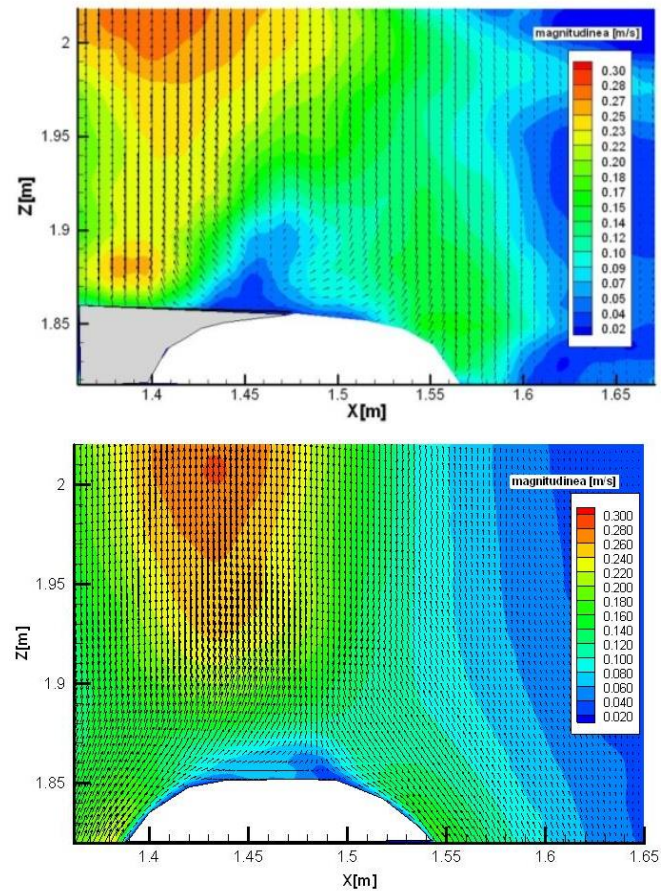

Fig. 6. Comparison between velocity fields with velocity vectors for natural convection case: Velocity magnitude $[\mathrm{m} / \mathrm{s}]$.

Fig. 5a indicates the points of measurement for longitudinal profile of temperature and extraction lines for velocity profiles and Fig. $6 \mathrm{~b}$ indicates the temperature fields obtained by IR technique.

In Fig 6. we have extracted velocity fields from experimental (PIV measurements) and numerical data. We can see a similar distribution above the head for both k-omega models, with same maximal values of velocity.

In Fig. 7 a) we superposed the variation of temperature above the manikin's head for the seven turbulence models comparing with experimental data obtained with two devices -IR thermography and a PT100 temperature probe. In Fig 7. b) and c) we superposed the variation of $\mathrm{U}$ and $\mathrm{W}$ velocity 
components for the same turbulence models comparing them with PIV measurements in sagittal plan.

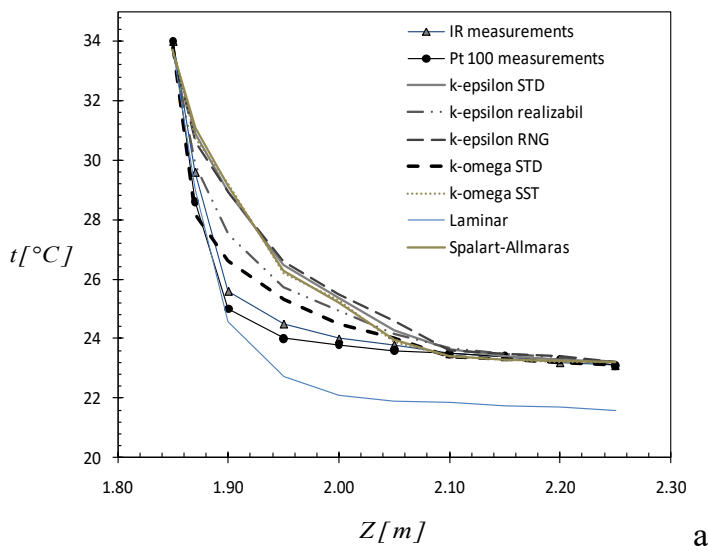

a)
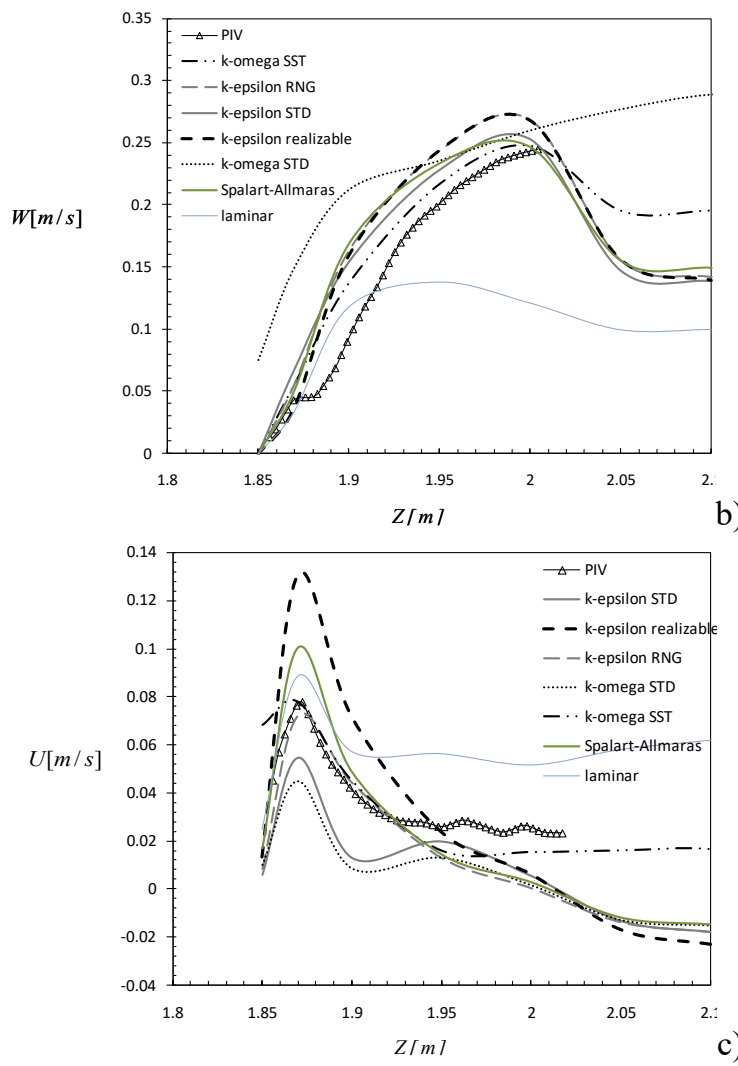

Fig. 7. Temperature variation (a) and velocity (b) and (c) above the head for the 7 turbulence models compared to experimental data;

In Fig. 7 we extracted the velocity components W and $U$ profiles at different heights in the sagittal plane of the PIV measurement field. These profiles were compared with corresponding velocity profiles of the seven turbulence models.

As we can see, in Fig. 7, SST k-omega viscous model is closest to experimental variations. In Fig. 8. we can see that the net benefit is preserved for velocity profiles at $\mathrm{Z}=1.90 \mathrm{~m}$, SST k-omega model is one that has two maximum points located approximately in the same positions for the $\mathrm{W}$ velocity component and an amplitude of the same order size for $\mathrm{U}$ component.
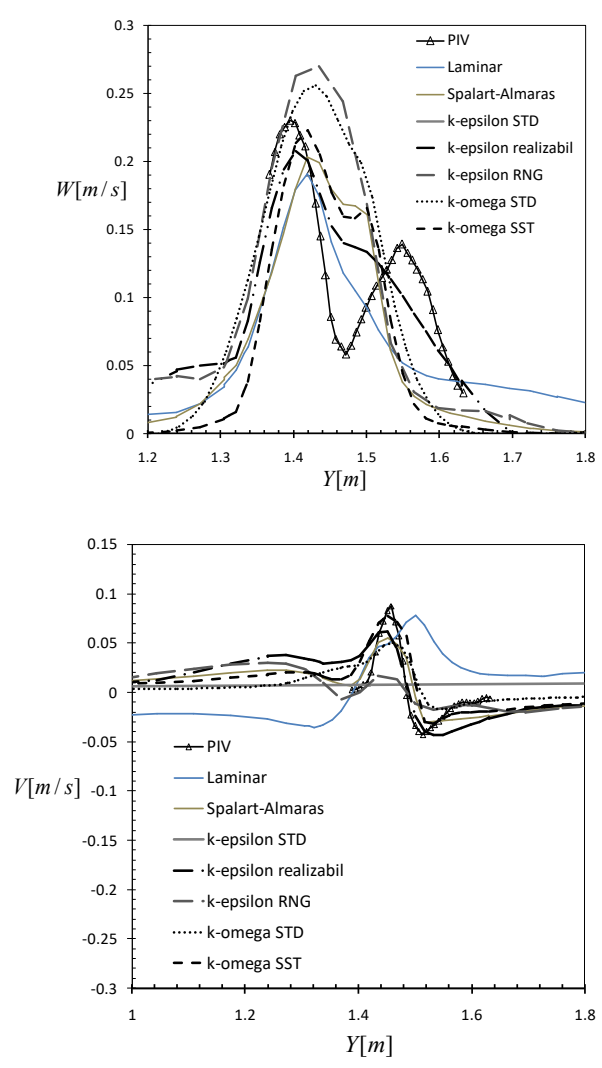

b)

Fig. 8. Transversal profiles for $\mathrm{W}$ and $\mathrm{V}$ component sagittal plan - comparison 7 turbulence models and PIV measurement: at $Z=1.90 \mathrm{~m}$

Reviewing the parameters used to choose the model, we obtain the general characteristics for the cases studied below. The study of grid dependency indicated us as satisfactory the case of 2.2 million cells, enough to obtain stable results. Viscous model, SST k-omega implemented for the organic geometry of the manikin is the one who found the best solution in agreement with experimental studies.

Because the human body releases heat in the first place by radiation was necessary to use a radiation model. Fluent solver proposes five models of radiation, the used one involves calculating form factors of each area involved in the radiation with the Surface to Surface model.

With the defined numerical model, we further used it for a preliminary study regarding the influence on Turbulence intensity on human body, when it has different values at the inlet boundary conditions.

\section{Results and discussion}

In this study we assessed the influence of turbulence intensity at inlet in the domain for piston ventilation case. This type of ventilation can be regarded as an extreme variant of the displacement ventilation system with a minimum of turbulence in the airflow through the room. Piston ventilation or variants can be found in special applications like clean rooms or operating theaters, for example. 
For the validated numerical model, different turbulence intensities were used for the boundary conditions in a preliminary simple case of the piston ventilation case. This type of inlet gives us the possibility to control more the turbulence intensity around the human body to extract the correlations between turbulence and thermal behavior. While the velocity fields are practically the same for all the studied case, we can see that in the case of the turbulence intensity we have large variations of thermal comfort studied parameters from one case to another.

The aim of this study is to evaluate the influence of turbulence intensity on thermal comfort. The inlet device is placed in front of the manikin and the outlet in the opposite part. Inlet velocity has a value $0.3 \mathrm{~m} / \mathrm{s}$, only varying the initial turbulence intensity: $5 \%, 10 \%, 20 \%$, $25 \%, 30 \%$ and $50 \%$. Piston ventilation implies supplying an airflow at low velocity resulting high air change rates.

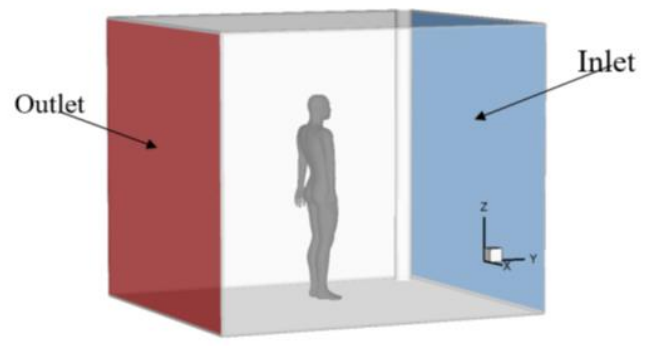

Fig. 9. Case studied: Piston type ventilation.

As it has been shown by Fanger [11], the velocity, temperature and the turbulence of the flow may generate a thermal discomfort translated by the sensation of "draught" as "an undesired cooling of the human body caused by air movement" $[11,12]$. This way, we wanted to check first, the influence of the variation of the inlet turbulence intensity on the behavior of the global temperature and velocity fields inside the test cell.

Therefore, in Fig. 10, we can see the temperature and velocity fields in a coronal and sagital plane passing through the virtual manikin for on a case, $\mathrm{Tu}=10 \%$, with the specification that the exact same temperature and velocity fields were obtained for all cases.
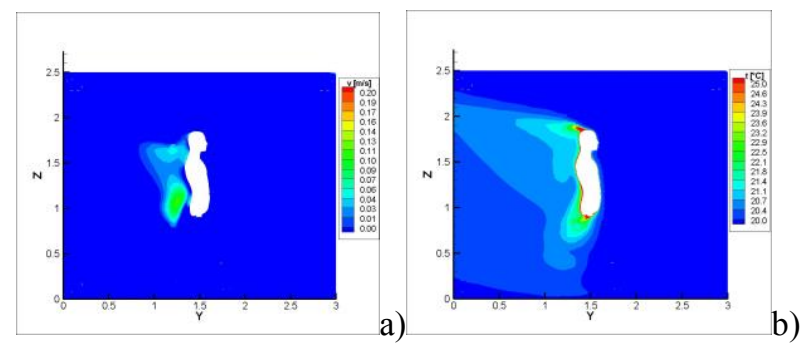

Fig. 10. Velocity and temperature fields for the case $\mathrm{Tu}=10 \%$

In Fig. 11 we can observe the turbulence intensity field in the manikin coronal plane for different turbulence intensities at the inlet.

While in the case without imposed flow, we could observe a relative stratification on the temperature field, in the cases with piston flow, the air surrounding the manikin has a coherent flow, allowing nevertheless the observation of the thermal plume. There is no noticeable difference between temperature or velocity fields corresponding to the cases with piston flow. However, there is a significant difference between the cases when considering coronal turbulence fields. As we can see in the next figure.

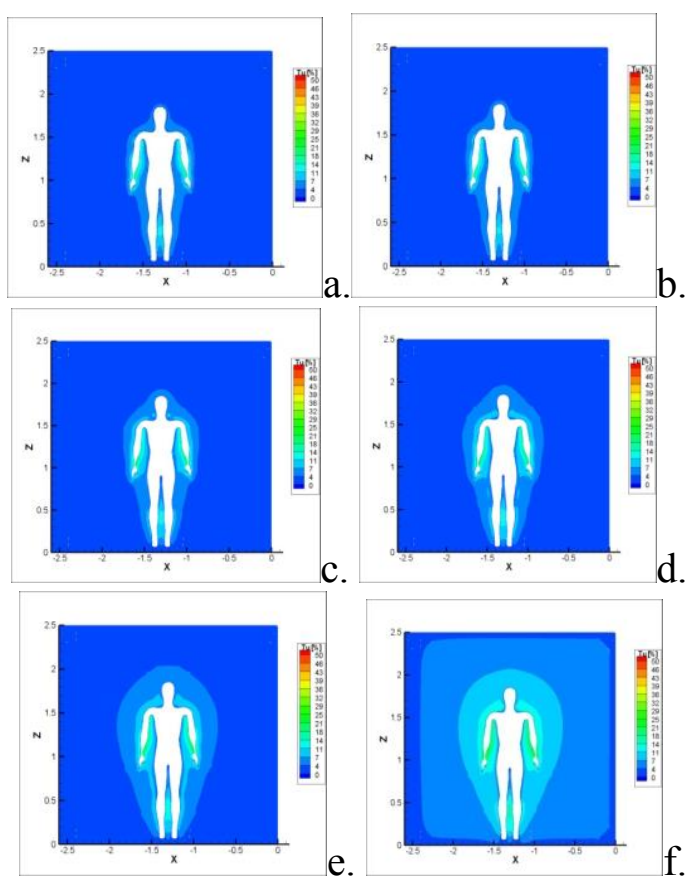

Fig. 11 Turbulence intensity field in the manikin coronal plane for different turbulence intensities at the inlet: $a .5 \%, b .10 \%, c$. $20 \%, d .25 \%, e .30 \%, f .50 \%$

The numerical simulation results allow us to evaluate the Draft Risk $(D R)$ index as defined by Fanger. While the PMV didn't reveal any changes between the cases, the DR index has shown different values around the human body. In the next figure is shown the corresponding distribution of the Draft rate $(D R)$. The spatial distributions of the $D R$ are divided in four main regions as indicated in the legend from the same figure. These four regions correspond to a classification of the indoor ambiance regarding its comfort level.

$$
D R=\left(34-t_{a}\right) \times\left(\bar{v}_{a}-0.05\right)^{0.62} \times\left(0.37 T_{u} \cdot \bar{v}_{a}+3.14\right)
$$

In wich:

$\mathrm{t}_{\mathrm{a}}$ - air mean temperature;

$\bar{v}_{a}$ - air mean velocity;
$\mathrm{T}_{\mathrm{u}}$ - turbulence intensity;

\begin{tabular}{|c|c|c|c|c|}
\hline \multicolumn{4}{|c|}{ INDOOR QUALITY } \\
\cline { 2 - 5 } & A & B & C & D \\
\hline DR & $<15 \%$ & $<20 \%$ & $<25 \%$ & $25 \%$ \\
\hline & HIGH & AVERAGE & LOW & INACCEPTABLE \\
\hline
\end{tabular}



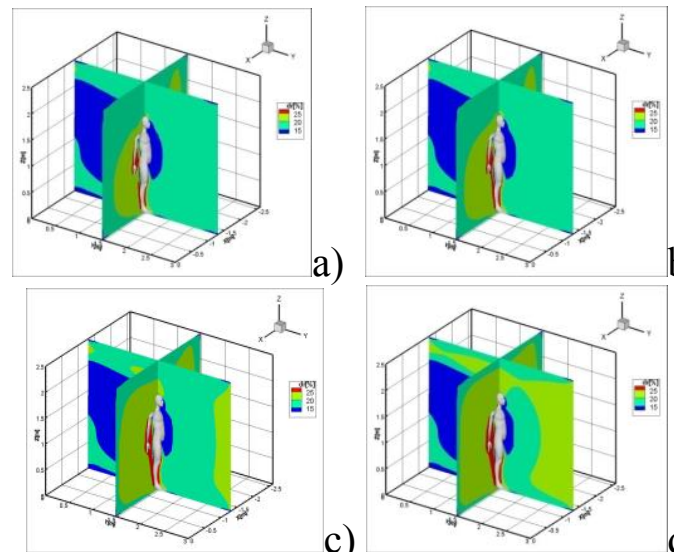

b)
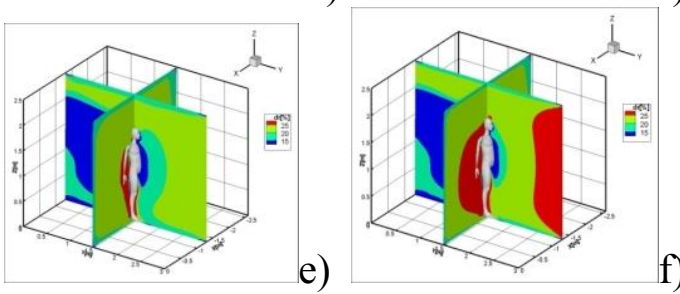

d)

Fig. 12. DR for the sagittal plane for the cases studied: $\mathrm{Tu}=$ a. $5 \%, b .10 \%$, c. $20 \%, d .25 \%$, e. $30 \%, f .50 \%$

The DR index indicates that the level of discomfort increases with the turbulence level imposed at the inlet, but it is mainly related to draft sensation. To better analyze the thermal comfort influence we have evaluated the convective heat loss for each case.

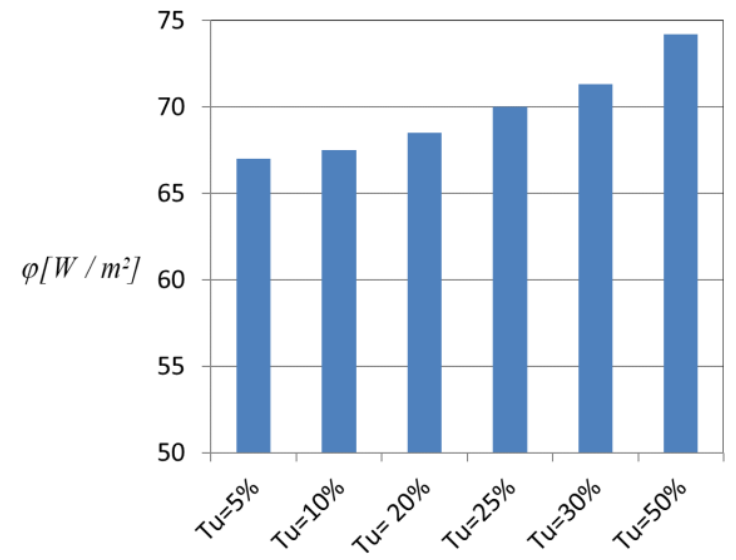

Fig. 13. Heat flux released by the human body correlated with the turbulence intensity at inlet level

The results showed that the turbulence intensity imposed at the inlet level has an important impact on the heat loss as we can see in Fig13. This correlation indicates that there a possible thermal comfort control when selecting a certain inlet for a distribution system.

\section{Conclusions}

The article presents a preliminary study regarding the inlet turbulence intensity on the state of the thermal comfort state. For the verification and validation of numerical model the natural convection case was chosen, without any imposed airflow at the inlet. We compared the velocity profiles overhead as well as global velocity and temperature fields. The SST k-omega viscous model has shown good results in comparison with experimental measurements. Moreover, we intended to evaluate the influence of turbulence intensity on thermal comfort and for this we have chosen a piston distribution system. The cases studied have different boundary conditions regarding the turbulence intensity: $5 \%, 10 \%, 20 \%, 25 \%$, $30 \%$ and $50 \%$, while the rest of the parameters were kept exactly the same. The preliminary studies have shown that there is a clear correlation between the turbulence intensity imposed at the inlet level, considering draft sensation but also thermal sensation. These results open the path for evaluating the influence of turbulence intensity on thermal comfort for other air distribution systems.

\section{Acknowledgements}

This work was supported by the grants: of the Romanian space agency ROSA, QUEST - Advanced air diffusion system of the crew quarters for the ISS and deep space habitation systems, STAR-CDI-C3-2016-577.

\section{References}

1. A. Mecke, I. Lee, J.R. Baker jr., M.M. Banaszak Holl, B.G. Orr, Eur. Phys. J. E 14, 7 (2004)

2. 1. Abadie, M., Contribution à l'étude de la pollution particulaire : rôle des parois, rôle de la ventilation, in LEPTAB. 2000, Université de La Rochelle.

3. 2. Fanger, P.O., ed. Thermal Comfort-Analysis and Applications in Environmental Engineering. ed. C.D.T. Press. 1970.

4. 3. Meslem, A., Contribution à l'étude du couplage thermique entre un jet et un local climatisé. 1997, INSA Lyon.

5. 4. Kosonen, R., et al., Impact of heat load location and strength on air flow pattern with a passive chilled beam system Energy and Buildings, 2010. 42(1): p. 34-42.

6. 5. Einberg, G., et al., CFD modeling of an industrial air diffuser-predicting velocity and temperature in the near zone. Building and Environment 2005. 40: p. 601-615.

7. 6. Meslem, A., et al., A comparison of three turbulence models for the prediction of parallel lobed jets in perforated panel optimization. Building and Environment, 2011. 46(11): p. 2203-2219.

8. 7. Inard, C., H. Bouia, and P. Dalicieux, Prediction of air temperature distribution in buildings with a zonal model. Energy and Buildings, 1996. 24(2): p. 125-132.

9. 8. Inard, C., P. Depecker, and J.-J. Roux, Un modèle simplifié pour la prédiction du champ de température dans les bâtimentsA simplified model for the prediction of air temperature distribution in 
buildings. Révue Générale de Thermique, 1997. 36(2): p. 113-123.

10. 9. Inard, C., et al., Structure moyenne et anlyse intégrale du panache thermique des convecteurs électriques. Révue Générale de Thermique, 1997. 36: p. 495-509.

11. 10. Awbi, H.B., Ventilation of Buildings. 2003, London, U.K.: E\&FN SPON. 313.

12. 11. Fanger, P.O., et al., Air turbulence and sensation of draught. Energy and Buildings, 1988. 12(1): p. 21-39.

13. 12. Fanger, P.O., The new comfort equation for indoor air quality. Ashrae Journal, 1989. 31(10): p. 33-38. 